\title{
Quantifying the Delocalization of Surface and Bulk F-Centers
}

\author{
Benjamin G. Janesko, ${ }^{\mathrm{a}, *}$, Stephanie I. Jones ${ }^{\mathrm{a}}$ \\ ${ }^{a}$ Department of Chemistry and Biochemistry, Texas Christian University, Fort Worth, Texas 76129, USA
}

\begin{abstract}
Electrons trapped in ionic crystal defects form color centers (F-centers) important in surface science, catalysis, and optoelectronic devices. We apply the electron delocalization range function (EDR) to quantify the delocalization of surface and bulk F-centers. The EDR uses computed one-particle density matrices to quantify "delocalization lengths" capturing the characteristic size of orbital lobes. Ab initio cluster model calculations confirm that the delocalization lengths of bulk alkali halide $F$-centers scale with the size of the anion vacancy. Calculations on magnesium oxide surface $F_{s}$ and $F_{s}^{+}$centers, as well as other anionic surface defects, show how the trapped electrons' delocalization depends on the defect morphology, defect occupancy, and the approximate treatment of electron correlation. Application to $\mathrm{N}_{2}$ activation by anionic surface defects illustrate how the trapped electron localizes into the adsorbed molecule's unoccupied orbitals. The results confirm that the EDR provides a useful tool for understanding the chemistry of surface- and bulk-trapped electrons.
\end{abstract}

Keywords: surface F-center, delocalization, electronic structure

\section{Introduction}

$F$-centers in alkali halides and alkaline-earth oxides have been studied for many decades[1, $2,3,4,5,6,7,8,9]$. F (Farbe) centers are defects in ionic crystals in which an anion is replaced by one or more trapped electrons. Confined and shielded by the surrounding crystal lattice, these trapped electrons form gap states possessing unique optical, electric, and magnetic properties[10, 11] relevant to optoelectronic devices[12, 13, 14, 15, 16]. Fcenters at surfaces, along with other anionic defects, are also relevant in catalysis[17, 18, 19, $20,21,22,23]$.

In this work, we use electronic structure calculations to study the quantum-mechanical localization of electrons trapped in surface and bulk $F$-centers[24, 25]. Electronic structure calculations simulating surface and bulk $F$-centers have a long history[7, 26, 27]. Modern simulations typically treat treat either a single $F$-center surrounded by a finite cluster of nearby atoms $[28,29,30,19,20,21,31]$, or an infinite, periodic array of more or less distant $F$-centers[32, 33, 23]. Many of these simulations target energetic properties such as

\footnotetext{
* Corresponding author.

Email address: b.janesko@tcu.edu (Benjamin G. Janesko)
}

Preprint submitted to Surface Science

January 13, 2017 
ionization or excitation energies. These properties can be quite sensitive to technical details of the simulation[33]. Fewer simulations consider our focus, the structure and localization of the trapped electrons. Previous studies of trapped electrons' localization consider either the single (highest occupied) orbital containing the trapped electron, $[34,35,36]$ or the spin polarization of the electron density[25]. Both approaches have limitations. Focus on single orbitals can be problematic for systems with "strong coupling", where molecular orbital theory breaks down.[37, 38] Anayses of spin polarization are not applicable to closed-shell systems, such as two singlet-coupled electrons trapped in a single defect. Bader and Platts went beyond these limitations, using the quantum theory of atoms in molecules[39] to characterize bulk $F$-centers in $\mathrm{LiF}[31]$. Calculations on a simple $\mathrm{Li}_{14} \mathrm{~F}_{12}^{+}$cluster model confirmed that the trapped electron yields a "non-nuclear attractor", a maximum in the electron density occuring not at a nucleus, but at the defect center. Non-nuclear attractors were also found in sodium electrosodalite[40] and magnesium oxide[41] F-centers. The electron localization function[42] also gives insight into F-centers[32]. However, none of these methods directly quantify the trapped electrons" "size", i.e., the quantum-mechanical delocalization length.

We recently proposed the electron delocalization range function $\operatorname{EDR}(\vec{r} ; d)$ to quantify and visualize aspects of electron delocalization[43]. The EDR quantifies the degree to which the one-particle density matrix

$$
\gamma\left(\vec{r}, \overrightarrow{r^{\prime}}\right)=N \int d^{3} \vec{r}_{2} \ldots \int d^{3} \vec{r}_{N} \Psi\left(\vec{r}, \vec{r}_{2} \ldots \vec{r}_{N}\right) \Psi^{*}\left(\vec{r}^{\prime}, \vec{r}_{2} \ldots \vec{r}_{N}\right)
$$

of calculated N-electron wavefunction $\Psi$ "delocalizes" distance $d$ about point $\vec{r}$. The EDR does this by projecting the density matrix onto a model density matrix $\gamma_{\text {mod }}$ which decays by construction over distance $d$ :

$$
\begin{aligned}
\operatorname{EDR}(\vec{r} ; d) & =\rho^{-1 / 2}(\vec{r}) \int d^{3} \overrightarrow{r^{\prime}} \gamma\left(\vec{r}, \overrightarrow{r^{\prime}}\right) \gamma_{\text {mod }}\left(\vec{r}-\overrightarrow{r^{\prime}}, d\right) \\
\gamma_{\text {mod }}(u, d) & =\left(\frac{2}{\pi u^{2}}\right)^{3 / 4} \rho^{1 / 2}(\vec{r}) \exp \left(-\frac{u^{2}}{d^{2}}\right)
\end{aligned}
$$

The prefactors in Eq. 3 ensure that $\gamma_{\text {mod }}$ has the proper units and normalization $\int d^{3} \overrightarrow{r^{\prime}}\left|\gamma\left(\vec{r}, \overrightarrow{r^{\prime}}\right)\right|^{2}=$ $\rho(\vec{r})$. The unitless EDR obeys $|\operatorname{EDR}(\vec{r} ; d)|^{2} \leq 1$ by the Cauchy-Schwarz inequality. Distance $D(\vec{r})=\operatorname{argmax}_{d} \operatorname{EDR}(\vec{r} ; d)$ maximizing $\operatorname{EDR}(\vec{r} ; d)$ at point $\vec{r}$ serves as a "characteristic delocalization length" of electrons at $\vec{r}$. The EDR is inspired by our[44] and others'[45, 46, 47] work quantifying delocalization of the one-particle density matrix and exchange hole. The EDR approximately quantifies the radius of the orbital lobes dominating in region $\vec{r}$ : for example, comparisons of the bonding and antibonding states of $\mathrm{H}_{2}^{+}$show that $D(\vec{r})$ is relatively large in the single lobe of the bonding orbital, and relatively small in each of the two lobes of the antibonding orbital[48]. (We use "orbital lobe" language as an aid to understanding, not because the EDR is in some way "orbital dependent". As a function of the full one-particle density matrix, the EDR is independent of unitary transforms of the occupied orbital space, and can readily be evaluated for multiconfigurational and multireference wavefunctions.) 
Our earliest study of the EDR considered a crude periodic supercell model of bulk $\mathrm{NaCl}$ $F$-centers. Points $\vec{r}$ in the defect site had $\operatorname{EDR}(\vec{r} ; d)$ maxima shifted to large $d[43]$.

The EDR provides a "characteristic delocalization length" $D_{\max }$ of an N-electron system's most weakly bound electron, defined as

$$
\begin{aligned}
D_{\text {max }} & =\operatorname{argmax}_{d}\langle\operatorname{EDR}(d)\rangle_{N}-\langle\operatorname{EDR}(d)\rangle_{N-1} \\
\langle\operatorname{EDR}(d)\rangle_{N} & =\int d^{3} \vec{r} \rho(\vec{r}) \operatorname{EDR}(\vec{r} ; d)
\end{aligned}
$$

Here $\langle\operatorname{EDR}(d)\rangle_{N}$ is the system-averaged EDR of an N-electron system, which is compared to the system average with N-1 electrons. The corresponding difference in total energies defines the vertical detachment energy (VDE). $D_{\max }$ of anionic water clusters, evaluated from Hartree-Fock calculations, has a one-to-one relationship with a very different measure of electron radius, the radius of gyration of the singly occupied molecular orbital[49, 35]. Real-space plots of $\operatorname{EDR}\left(\vec{r} ; D_{\max }\right)$ characterize the region of space occupied by the trapped electron, highlighting the major lobe of the singly occupied orbital. Evaluating the EDR from correlated wavefunctions quantifies the effects of "strong" correlation on the electron distribution[49, 50]. Our most recent study used $D_{\max }$ to quantify strong correlation in coupled F-center defects, which we showed map onto the to the "textbook" problem of strong correlation in stretched $\mathrm{H}_{2}[51]$.

Here we significantly extend Ref. [51] by applying the EDR to surface and bulk Fcenters and other anionic defects. Our results confirm that the EDR is a practical tool for building insight into trapped electrons. We find that in alkali halide bulk $F$-centers, the trapped electrons' $D_{\max }$ scales with the size of the cavity. Calculations on surface F-centers and other surface defects on magnesium oxide confirm the relatively large delocalization of electrons trapped at surface defects. Application to $\mathrm{N}_{2}$ activation by surface trapped electrons[28] illustrate how the trapped electron localizes onto the absorbed molecule.

\section{Computational Methods}

Calculations use the development version of the Gaussian suite of programs[52]. Calculations use Hartree-Fock theory (HF), second-order many-body perturbation theory (MP2), and generalized Kohn-Sham density functional theory (DFT) [53, 54]. DFT calculations use several density functional approximations (DFAs) for the exact exchange-correlation functional: the local spin-density approximation (LSDA)[55], the Perdew-Burke-Ernzerhof generalized gradient approximation (PBE GGA)[56], and Becke's three-parameter global hybrid incorporating Lee-Yang-Parr correlation (B3LYP) [57, 58, 59]. Most calculations use the $6-31+\mathrm{G}(\mathrm{d})$ atom-centred basis set, freeze the atomic positions to those of the experimental defect-free bulk cubic lattice, and place "ghost" atom basis functions at the defect center. Calculations on open-shell systems are performed spin-unrestricted. The EDR and $D_{\max }$ are evaluated as described previously[43]. Calculations on the particle-in-a-box model use a Mathematica worksheet provided as Supporting Information. Calculations comparing ground and excited states use the experimental lattice parameters and embed the clusters in $>100$ effective core potentials and $>1000$ point charges to simulate the surrounding ionic 

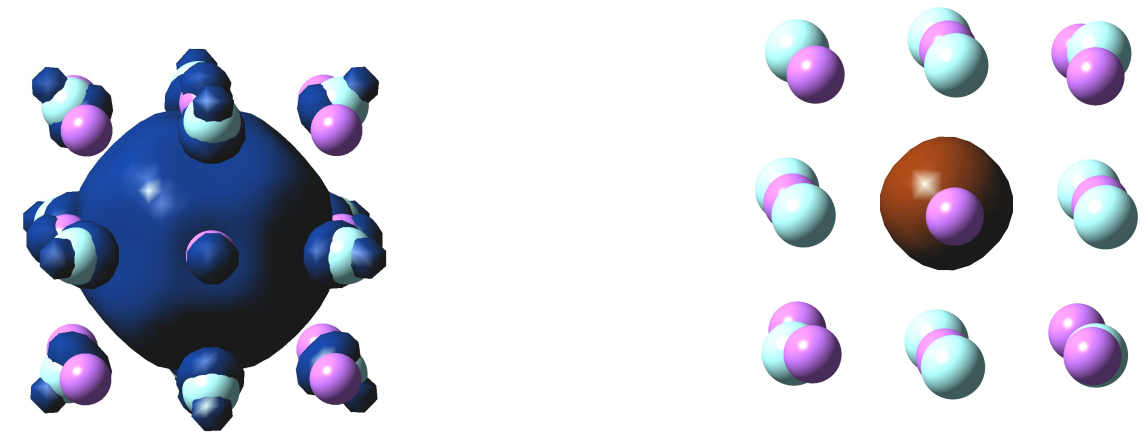

Fig. 1. Isosurfaces of spin density (left) and $\operatorname{EDR}\left(\vec{r} ; D_{\max }\right)$ (right) for a cluster model of an isolated bulk LiF F-center.

lattice. As in Ref. [51], we simulate $\mathrm{Li}^{+}$and $\mathrm{F}^{-}$as point charges +1 and -1 surrounded by the SBKJC[60] large-core effective core potentials for $\mathrm{Li}^{+}$and $\mathrm{Na}^{+}$, respectively.

\section{Results and Discussion}

\subsection{Bulk F-centers}

Before considering surface $F$-centers, we confirm that the EDR gives reasonable results for bulk $F$-centers. Table 1 reports $D_{\max }$ evaluated for isolated paramagnetic bulk $F$-centers in alkali halides. $D_{\max }$ is compared to the cavity radius $L$, defined as the nearest-neighbor distance of the defect-free crystal at $0 \mathrm{~K}[61]$. $D_{\max }$ is evaluated for the cluster model in Figure 1, and for a much simpler model[62]: a single electron in a cubic box of side length $2 L$. Numerical evaluation yields the linear relation

$$
D_{\max }=0.845 \mathrm{~L}
$$

(Supporting Information). This physically realistic result shows that the trapped electron's characteristic radius $D_{\max }$ is somewhat less than the cavity radius $L$. The more realistic $a b$ initio cluster model gives a somewhat smaller $D_{\max }$, consistent with Pauli repulsion from the cavity wall. Figure 1 shows that $\operatorname{EDR}\left(\vec{r} ; D_{\max }\right)$ highlights the major lobe of the electron spin density, consistent with the EDR from electrons trapped in solvent clusters[49] and electrides[50].

Bare clusters like those in Figure 1 provide a rather poor model of the energetics of $F$ center defects[33]. We thus investigate how well the bare cluster models the defect electronic structure, comparing electrostatic embedding's effects on VDE vs. $D_{\max }$. Table 2 reports the VDE and $\mathrm{D}_{\max }$ of an isolated bulk paramagnetic $F$-center in $\mathrm{NaCl}$, evaluated for a $\mathrm{Na}_{14} \mathrm{Cl}_{13}^{+}$cluster embedded in $N_{P C}$ shells of point charges. (We choose even $N_{P C}$ to ensure a 
Table 1

Cavity radius $L$ and delocalization length $D_{\max }$ of bulk alkali halide $F$-centers.

\begin{tabular}{lccc}
\hline \hline Property & $\mathrm{LiF}$ & $\mathrm{NaCl}$ & $\mathrm{KBr}$ \\
\hline Cavity radius $L$ (Bohr, Ref. [61] & 3.81 & 5.33 & 6.23 \\
$D_{\max }$ (bohr), Eq. 6 & 3.22 & 4.50 & 5.26 \\
$D_{\max }$ (bohr), cluster model & 2.81 & 4.08 & 4.47 \\
\hline
\end{tabular}

Table 2

VDE and $D_{\max }$ for $\mathrm{Na}_{14} \mathrm{Cl}_{12}^{+}$model of $\mathrm{NaCl} F$-center, embedded in $N_{P C}$ shells of point charges.

\begin{tabular}{lrr}
\hline \hline$N_{P C}$ & VDE (eV) & $D_{\max }($ Bohr $)$ \\
\hline 0 & 5.32 & 4.2 \\
2 & 5.17 & 4.2 \\
4 & 4.87 & 4.2 \\
6 & 4.72 & 4.2 \\
8 & 4.64 & 4.2 \\
10 & 4.59 & 4.2 \\
12 & 4.55 & 4.2 \\
\hline \hline
\end{tabular}

total cluster charge +1$)$. Electrostatic embedding has a dramatic effect on the VDE, but a negligible effect on $D_{\max }$, confirming that confinement is largely a function of the first shell of surrounding ions.

Our previous studies of the EDR highlighted how it characterizes the size of individual orbital lobes[48]. Accordingly, we expect that the ${ }^{2} S \rightarrow{ }^{2} P$ excited state of an alkali halide $F$-center would have $D_{\max }$ significantly smaller than the ground-state, due to the presence of two orbital lobes and an internal node in the excited orbital. Our previous study[51] concurred with other work[33] that excited-state calculations on cluster models require embedding potentials to prevent the excited electron from delocalizing onto the cluster surface. Accordingly, we perform a TDUHF/cc-pVDZ calculation on $\mathrm{Li}_{14} \mathrm{~F}_{12}^{+}$embedded in a combination of point charges and large-core ECPs following our earlier study[51]. The computed excitation energy $6.1 \mathrm{eV}$ is consistent with previous Hartree-Fock studies, overestimating the 5.0-5.1 eV seen experimentally[33]. We find that $D_{\max }$ changes from $2.56 \mathrm{Bohr}$ in the ground state to 1.70 Bohr in the first excited state, consistent with the smaller p-type orbital lobes occupied in the excited state (cf. Ref. [33] Figure 2).

The ongoing interest in Mollow-Ivey relations between $\mathrm{F}$ centers' lattice constant and absorbance spectra[63] motivates systematic explorations of the relation between defect radius and trapped electron size. Ref. [63] characterized this relation by evaluating the position of the first radial node $r_{0}$ in the singly occupied molecular orbital (SOMO) containing the trapped electron, for a cluster model of the $\mathrm{LiF} F$-center in which the lattice parameter $L$ 


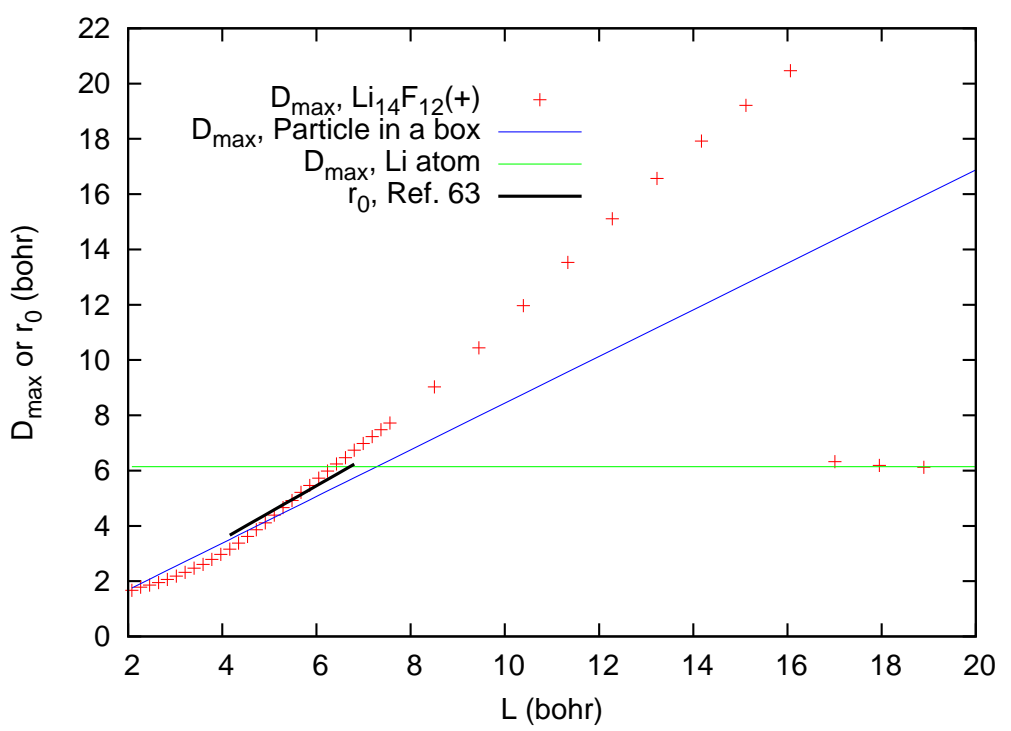

Fig. 2. $D_{\max }$ vs. cavity size $L$ for $\mathrm{LiF} F$-center defect. (Red points) $\mathrm{Li}_{14} \mathrm{~F}_{12}^{+}$cluster model. (Blue line) Particle in a box model of Eq. 6. (Green horizontal line) Isolated Li atom. (Thick black line) Orbital radius $r_{0}$ of Ref. [63].

is rigidly shifted. We have shown that for anionic water clusters, $D_{\max }$ from Hartree-Fock calculations has a one-to-one correspondence with the SOMO radius of gyration[49, 35]. Here we consider Mollow-Ivey type relations between $D_{\max }, r_{0}$, and $L$. Figure 2 plots $D_{\max }$ and $r_{0}$ vs. $L$ from $\mathrm{UHF} / 6-31+\mathrm{G}(\mathrm{d}, \mathrm{p})$ calculations on a cubic $\mathrm{Li}_{14} \mathrm{~F}_{12}^{+}$cluster with cavity radius $L$. Results are compared to the particle-in-a-box model of Eq. 6, and to the $r_{0}$ of Ref. [63]. Gratifyingly, all three measures of the trapped electron's size agree. The cluster and particle-in-a-box $D_{\max }$ are quite close to each other at small lattice parameter $L$, and lie close to the fit $r_{0}(L)=\alpha\left(L-c_{0}\right)$ in Ref. [63].

It is worth commenting on the cluster model results at large $L$. In this limit, the cluster behaves approximately as isolated $\mathrm{Li}$ and $\mathrm{F}$ atoms. Computed Mulliken populations show that the spin density is mostly delocalized over the six Li atoms nearest the cavity center. Thus $D_{\max }$ at large $L$ equals that of an isolated Li atom (Figure 2 horizontal line). The discontinuity in $D_{\max }$ after $L=17$ bohr is simply a consequence of the maximization in Eq. 4. At large $L$, the right-hand side of Eq. 4 has two maxima around $d=6.3$ and $d=20$ bohr. The large- $d$ maximum is the global maximum up to $L=16 \mathrm{bohr}$, while the small- $d$ maximum becomes the global maximum at larger $L$.

\subsection{Alkali halide surface $\mathrm{F}$-centers}

We next consider $F_{s}$ centers at alkali halide surfaces[64]. Previous studies predict that alkali halide $F_{s}$ centers have smaller transition energies than bulk centers, consistent with weaker binding of the trapped electron[65]. We model the $\mathrm{LiF} F_{s}$ center as a neutral $\mathrm{Li}_{9} \mathrm{~F}_{8}$ cluster with an open top. Calculations follow Figure 1, treating the isolated cluster at the $\mathrm{UHF} / 6-31+\mathrm{G}(\mathrm{d}, \mathrm{p})$ level and freezing the atoms at the experimental bulk lattice parameters. 

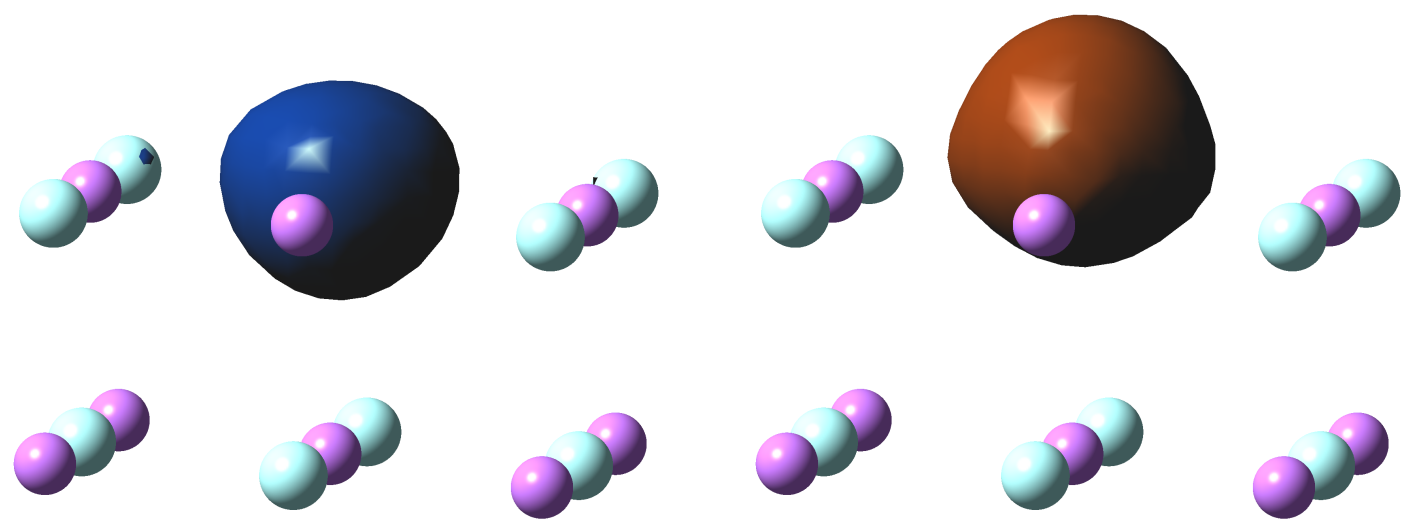

Fig. 3. Isosurfaces of spin density (left) and $\operatorname{EDR}\left(\vec{r} ; D_{\max }\right)$ (right) for $\mathrm{Li}_{9} \mathrm{~F}_{8}$ cluster model of surface $F_{s}$-center.

Figure 3 shows the computed structure, spin density, and $\operatorname{EDR}\left(\vec{r} ; D_{\max }\right)$ from the isolated $\mathrm{Li}_{9} \mathrm{~F}_{8}$ cluster. Again, the EDR clearly highlights the largest lobe of the spin density, showing the most delocalized electron trapped at the surface. $D_{\max }$ changes from $2.81 \mathrm{bohr}$ in the bulk $F$-center to 3.41 bohr in the surface $F_{s}$ center, consistent with a more weakly bound trapped electron.

One useful property of the EDR is that it may be evaluated from any level of theory that produces a one-particle density matrix $[49,50]$. Here we consider how basis set and electron correlation change the delocalization of surface and bulk $F$-centers. Table 3 reports $D_{\max }$ evaluated for the $\mathrm{Li}_{14} \mathrm{~F}_{12}^{+}$and $\mathrm{Li}_{9} \mathrm{~F}_{8}$ clusters simulating lithium fluoride bulk and surface $F$-centers. Calculations use the experimental lattice parameter and the cc-pVDZ or cc-pVTZ basis sets. We compare unrestricted Hartree-Fock calculations to restricted openshell Hartree-Fock; MP2; and DFT with the LSDA, PBE, and B3LYP exchange-correlation functionals. Triple-zeta MP2 calculations were unavailable due to memory limitations.

The results in Table 3 show that the $F$-center delocalization is relatively insensitive to the level of theory employed. Improving the basis set gives a lower total energy in these variational calculations, leading to a more tightly bound and more localized trapped electron. MP2 correlation allows the trapped electron to closer approach the cavity walls, giving a modest increase in $D_{\max }$ from UHF to MP2. Density functional theory calculations tend to "over-delocalize" the s-type orbital lobe containing the bound electron. This is consistent with previous evidence of DFT delocalization error[66, 67], though we note that the orbital lobe size measured by the EDR is not the same as the delocalization of charge or spin over multiple centers.

\subsection{Magnesium oxide surface F-centers}

Electrons at metal oxide surfaces are reactive species implicated in much surface chemistry[68]. Oxygen vacancies on magnesium oxide surfaces can trap one or two electrons, giving para- 


\section{Table 3}

$D_{\max }$ (bohr) for LiF bulk and surface F-centers. Results for five methods evaluated in double- and triple-zeta basis sets.

\begin{tabular}{lrrrr}
\hline \hline & \multicolumn{2}{c}{ Bulk } & \multicolumn{2}{c}{ Surface } \\
Method & DZ & TZ & DZ & TZ \\
\hline UHF & 2.88 & 2.81 & 3.46 & 3.55 \\
MP2 & 2.96 & - & 3.69 & - \\
B3LYP & 3.09 & 2.97 & 4.04 & 3.75 \\
PBE & 3.11 & 3.00 & 4.09 & 3.78 \\
LSDA & 3.09 & 2.95 & 4.09 & 3.76 \\
\hline \hline
\end{tabular}

\section{Table 4}

$D_{\text {max }}$ (bohr) of $\mathrm{MgO} F_{s}^{+}$(one trapped electron) and $F_{s}$ (two trapped electron) defects at terraces, edges, and corners. Results for the bare and $\mathrm{N}_{2}$-bound reverse corner defect $\mathrm{MgO}\left(\mathrm{e}^{-}\right)\left(\mathrm{H}^{+}\right)$(Section 3.4) are included for comparison.

\begin{tabular}{lrr}
\hline \hline System & One trapped electron & Two trapped electrons \\
\hline Surface & 2.76 & 3.25 \\
Edge & 3.00 & 3.71 \\
Corner & 3.33 & 4.50 \\
$\mathrm{MgO}\left(\mathrm{e}^{-}\right)\left(\mathrm{H}^{+}\right)$ & 3.39 & - \\
$\mathrm{MgO}\left(\mathrm{e}^{-}\right)\left(\mathrm{H}^{+}\right)-\mathrm{N}_{2}$ & 3.39 & - \\
\hline \hline
\end{tabular}

magnetic $F_{s}^{+}$and diamagnetic $F_{s}$ defects. As discussed above, the EDR is particularly well suited to characterizing diamagnetic defects where the electron spin density is not available. Pacchioni and coworkers have extensively studied the structure of $F_{s}$ and $F_{s}^{+}$defects on magnesium oxide terraces, edges, and corners[25, 69, 70]. Computed charge and spin densities show that the trapped electrons tend to reside in the vacancy[70]. The rather large formation energies of oxygen vacancies are somewhat reduced at low-coordinated edge and corner sites, making them potentially more experimentally relevant[25].

Figure 4 shows the structures, highest occupied molecular orbital (HOMO), and $\operatorname{EDR}\left(\vec{r} ; D_{\max }\right)$ for diamagnetic $F_{s}$ defects $\mathrm{MgO}$ terraces, edges, and corners. Table 4 reports the $F_{s}$ and $F_{s}^{+} D_{\max }$. These HF/6-31+G(d,p) calculations use the cluster models of Refs. [25, 69] embedded in an array of point charges \pm 2 simulating the Madelung potential. Atomic positions are frozen at the experimental bulk lattice parameter. The results are chemically reasonable: $D_{\max }$ is larger for the less coordinated edge and corner defects, and larger when two rather than one electrons are trapped in the surface defect. Figure 4 shows again that $\operatorname{EDR}\left(\vec{r} ; D_{\max }\right)$ highlights the major lobe of the frontier orbitals, showing the electron trapped in the defect. 

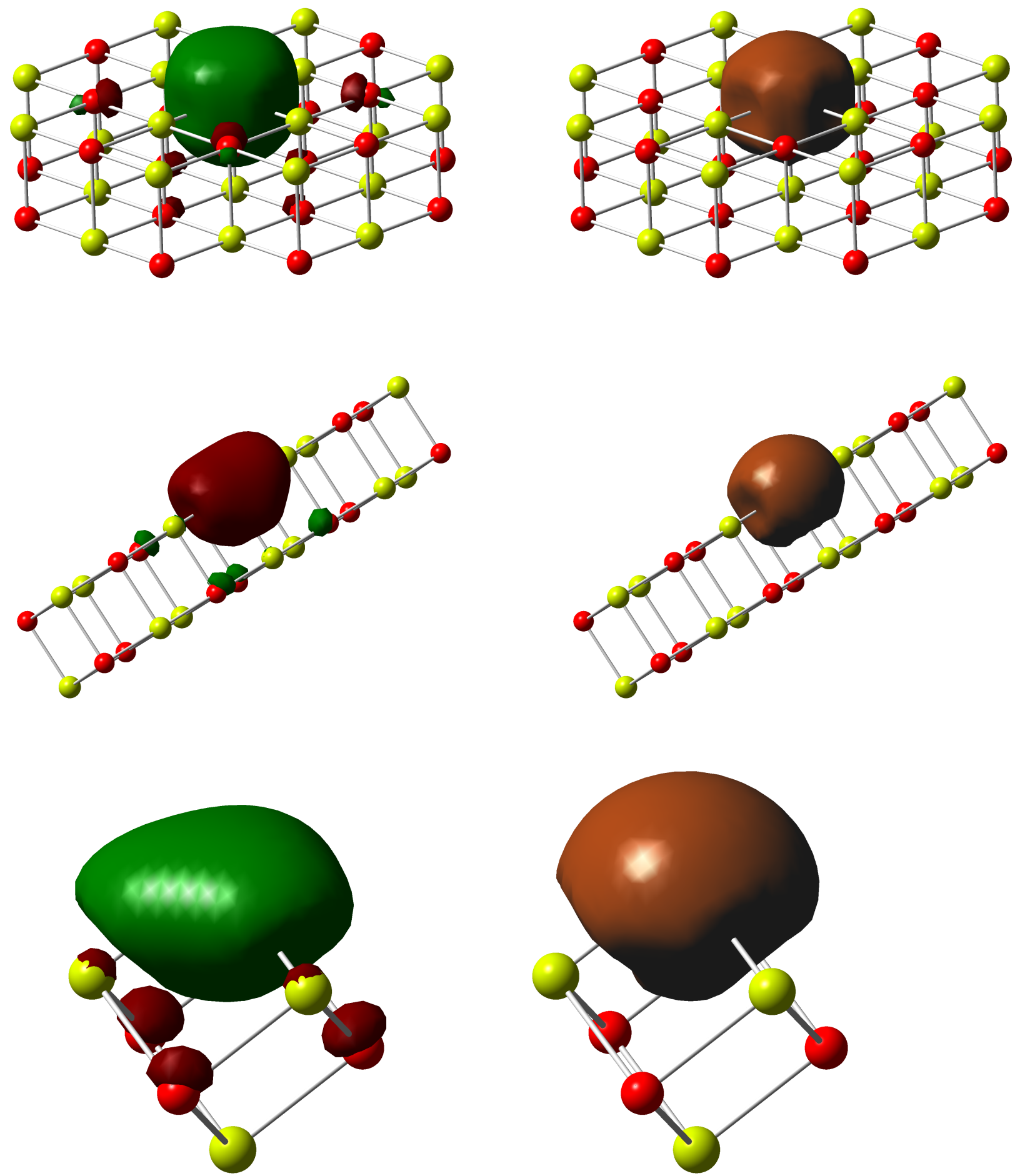

Fig. 4. Diamagnetic $F_{s}$ centers at the $\mathrm{MgO}$ surface. Isosurfaces of the HOMO $\left|\psi_{\text {HOMO }}(\vec{r})\right|=0.05 \mathrm{bohr}^{-3 / 2}$ (left) and $\operatorname{EDR}\left(\vec{r} ; D_{\max }\right)=0.7$ (right) for terrace, edge, and corner $F_{s}$ centers. 
Table 5

$D_{\max }$ (bohr) of $\mathrm{MgO} F_{s}^{+}$corner defects, evaluated at different levels of theory.

\begin{tabular}{lrr}
\hline \hline Method & DZ & TZ \\
\hline HF & 3.54 & 3.43 \\
MP2 & 3.48 & 3.41 \\
CCSD & 3.53 & - \\
B3LYP & 3.57 & 3.44 \\
PBE & 3.50 & 3.40 \\
LSDA & 3.57 & 3.44 \\
\hline
\end{tabular}

Calculations on the small, relatively weakly bound corner $F_{s}^{+}$defect further highlight the role of electron correlation. Table 5 shows $D_{\max }$ of the corner $F_{s}^{+}$center evaluated at several levels of theory. Improving the basis set localizes the $F_{s}^{+}$center consistent with Table 3. MP2 correlation makes the electrons more localized and more tightly bound. The more accurate CCSD method corrects MP2 over-localization. The DFT methods tend to over-delocalize the trapped electrons, particularly in the larger triple-zeta basis set.

\subsection{Other paramagnetic defects at magnesium oxide surfaces}

Pacchioni and coworkers have proposed an alternative model for paramagnetic color centers at $\mathrm{MgO}$ surfaces. Noting the large formation energies of oxygen defects, and the presence of EPR coupling between the surface trapped electron and an adjacent $\mathrm{H}$ nuclide, they suggest that the paramagnetic color centers are $\mathrm{MgO}\left(\mathrm{e}^{-}\right)\left(\mathrm{H}^{+}\right)$defects where an electron trapped at a low-symmetry site interacts with an adjacent $\mathrm{H}^{+}[28]$.

Figure 5 shows the computed spin density and EDR for one such defect, an electron trapped at a $\mathrm{MgO}$ reverse corner with an adjacent $\mathrm{H}^{+}$. These Hartree-Fock/6-31+G(d,p) calculations treat a neutral $\mathrm{Mg}_{14} \mathrm{O}_{14} \mathrm{H}$ cluster embedded in $>500$ point charges \pm 2 simulating the surrounding lattice. Calculations freeze the $\mathrm{Mg}$ and $\mathrm{O}$ atoms to experimental bulk lattice positions and optimized the $\mathrm{H}^{+}$position. $D_{\max }$ are reported in Table 4 above. Again, we find that $\operatorname{EDR}\left(\vec{r} ; D_{\max }\right)$ highlights the position of the major lobe of the spin density. The adjacent $\mathrm{H}^{+}$reduces $D_{\max }$ to a value comparable to the corner $F_{s}^{+}$defect, further confirming that these defects are chemically reasonable.

\subsubsection{Charge transfer to adsorbed $N_{2}$}

We conclude by considering the chemical reactivity of trapped electrons. Paramagnetic defects at $\mathrm{MgO}$ surfaces are known to be quenched by gas adsorption, with the characteristic EPR signal of the trapped electron disappearing[70, 71, 72]. We here consider one model for such quenching explored in Ref. [72], $\mathrm{N}_{2}$ adsorption to the reverse corner $\mathrm{MgO}\left(\mathrm{e}^{-}\right)\left(\mathrm{H}^{+}\right)$. Calculations use the model in Figure 5 with the $\mathrm{N}$ and $\mathrm{H}$ atom positions optimized. Figure 6 shows the computed spin density and $\operatorname{EDR}\left(\vec{r} ; D_{\max }\right)$. Table 4 shows that $D_{\max }$ is further reduced to $2.18 \mathrm{bohr}$, comparable to the 1.98 bohr seen for an isolated $\mathrm{N}_{2}^{-}$anion 

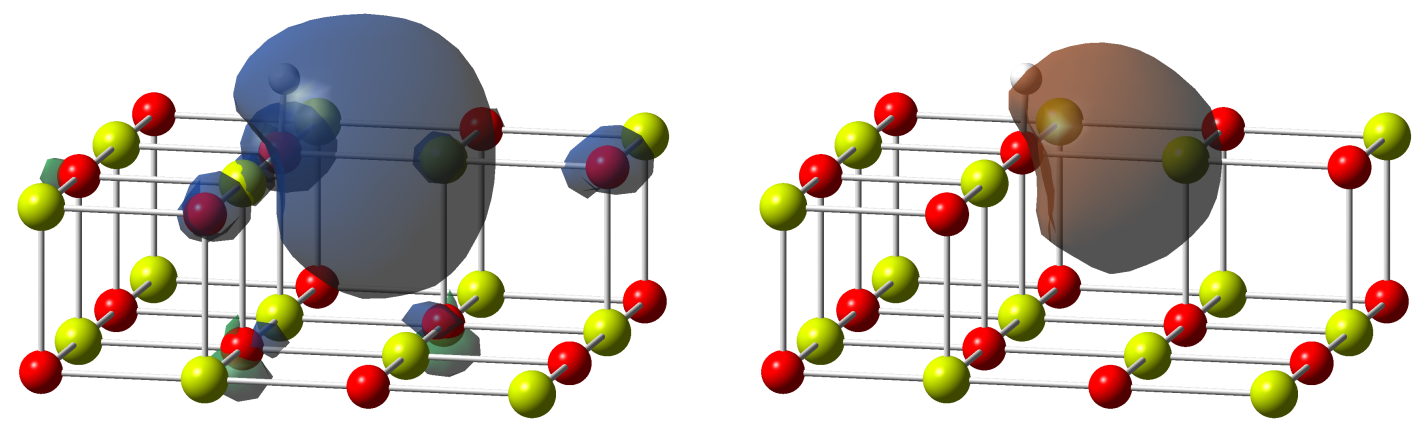

Fig. 5. Isosurfaces of spin density (left) and $\operatorname{EDR}\left(\vec{r} ; D_{\max }\right)$ (right) for $\mathrm{MgO}\left(\mathrm{e}^{-}\right)\left(\mathrm{H}^{+}\right)$reverse corner defects.
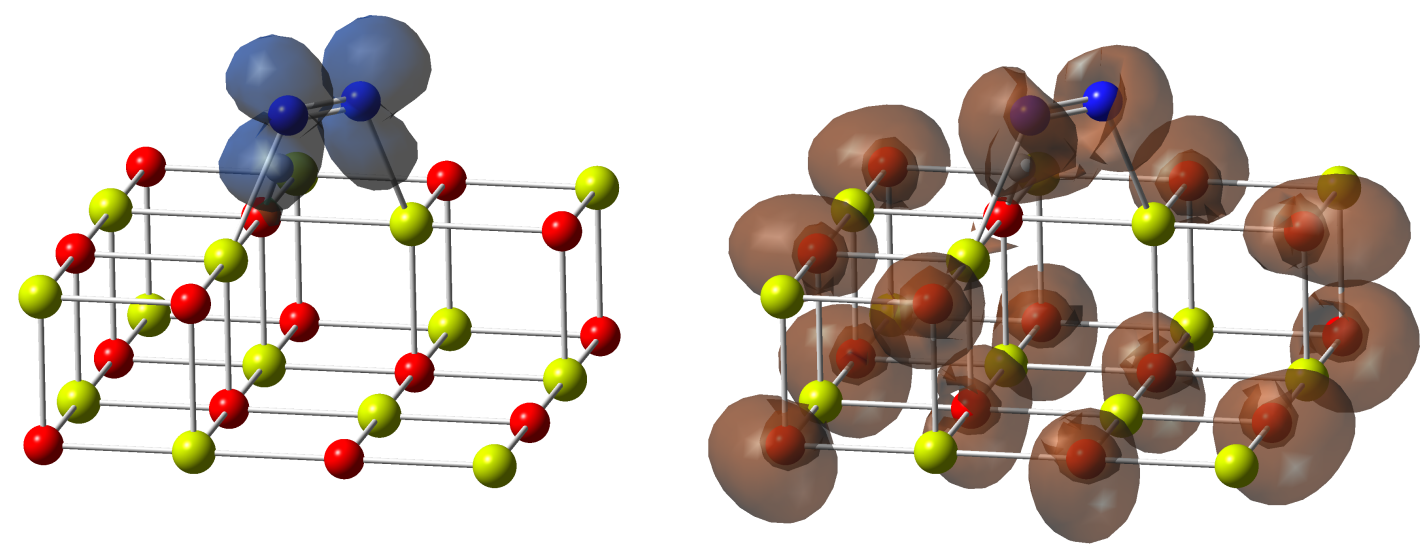

Fig. 6. As in Figure 5, for $\mathrm{N}_{2}$ adsorbed to the defect. 
at this level of theory. Figure 6 confirms that the trapped electron is almost completely transferred to the adsorbed $\mathrm{N}_{2}$. The electron resides in an antibonding orbital, giving a small $D_{\max }$ consistent with that seen for other antibonding orbitals[48]. Unlike the cases studied above, this $D_{\max }$ is in the range of characteristic delocalization lengths of the $\mathrm{O}^{2-}$ valence shell. $\operatorname{EDR}\left(\vec{r} ; D_{\max }\right)$ thus shows significant contributions from the $\mathrm{MgO}$ lattice, as well as the trapped electron. Overall, our results show that the EDR effectively maps out the consequences of gas adsorption for the trapped electron.

\section{Conclusion}

This work demonstrates that the electron delocalization range EDR is a useful interpretive tool for characterizing surface and bulk $F$-centers and other surface trapped electrons. Bulk $F$-centers' characteristic delocalization length $D_{\max }$ scales with defect size (Eq. 6) and maps out Mollow-Ivey-type relations. The density-matrix-based EDR can characterize $a b$ initio calculations and diamagnetic defects, where analyses of electron spin densities or single molecular orbitals provide an incomplete picture. Plots of $\operatorname{EDR}\left(\vec{r} ; D_{\max }\right)$ clearly highlight the locations of trapped electrons, and highlight trapped electrons' reactivity (Figures 5-6). Overall, the EDR shows significant potential for studies of more complex defect chemistry.

\section{Acknowledgments}

This material is based on work supported by the National Science Foundation under Grant No. DMR-1505343. The authors acknowledge the TCU High-Performance Computing Center for providing HPC resources.

\section{References}

[1] N. Itoh, Creation of Lattice Deects by Electronic Excitation in Alkali Halides, Adv. Phys. 31 (1982) 491-551.

[2] N. Itoh, K. Tanimura, Formation of Interstitial-Vacancy Pairs by Electronic Excitation in Pure IonicCrystals, J. Phys. Chem. Solids 51 (1990) 717-735.

[3] R. T. Williams, K. S. Song, The Self-Trapped Exciton, J. Phys. Chem. Solids 51 (1990) 679-716.

[4] R. T. Williams, K. S. Song, Self-Trapped Excitons, Springer, Berlin, 1993.

[5] N. W. Lord, Electron-Nuclear Double Resonance of F Centers in Lithium Fluoride, Physical Review Letters 1 (1958) 170-171.

[6] F. Seitz, Color Centers in Alkali Halide Crystals. II, Rev. Mod. Phys. 26 (1954) 7-94.

[7] F. Seitz, Color centers in alkali halide crystals, Reviews of Modern Physics 18 (1946) 384-408.

[8] W. T. Doyle, Optical Absorption by F Centers in Alkali Halides, Physical Review 111 (1958) 1072-1077.

[9] W. C. Holton, H. Blum, Paramagnetic resonance of $\mathrm{f}$ centers in alkali halides, Physical Review 125 (1962) 89-103.

[10] J. J. Markham, F-centers in Alkali Halides, Academic Press, New York, 1966.

[11] A. M. Stoneham, Theory of Defects in Solids, Clarendon Press, Oxford, 1975.

[12] S.-J. Yu, M.-W. Kang, H.-C. Chang, K.-M. Chen, Y.-C. Yu, Bright fluorescent nanodiamonds: No photobleaching and low cytotoxicity, Journal of the Americal Chemical Society 127 (50) (2005) 1760417605 . 
[13] A. L. Falk, P. V. Klimov, B. B. Buckley, V. Ivády, I. A. Abrikosov, G. Calusine, W. F. Koehl, A. Gali, D. D. Awschalom, Electrically and mechanically tunable electron spins in silicon carbide color centers, Phys. Rev. Lett. 112 (2014) 187601.

[14] V. Jacques, E. Wu, F. Grosshans, F. Treussart, P. Grangier, A. Aspect, J.-F. Roch, Experimental realization of wheeler's delayed-choice gedanken experiment, Science 315 (5814) (2007) 966-968.

[15] W. F. Koehl, B. B. Buckley, F. J. Heremans, G. Caulsine, D. D. Awschalom, Room temperature coherent control of defect spin qubits in silicon carbide, Nature 479 (2011) 84-88.

[16] L. C. Bassett, F. J. Heremans, D. J. Christle, C. G. Yale, G. Burkard, B. B. Buckley, D. D. Awschalom, Ultrafast optical control of orbital and spin dynamics in solid-state defect, Science 345 (6202) (2014) $1333-1337$.

[17] B. Yoon, H. Häkkinen, U. Landman, A. S. Wörz, J.-M. Antonetti, S. Abbet, K. Judai, U. Heitz, Charging Effects on Bonding and Catalyzed Oxidation of $\mathrm{CO}$ on $\mathrm{Au}_{8}$ Clusters on $\mathrm{MgO}$, Science 307 (2005) 403-407.

[18] G. Pacchioni, S. Sicolo, C. D. Valentin, M. Chiesa, E. Giamello, A Route toward the Generation of Thermally Stable Au Cluster Anions Supported on the MgO Surface, Journal of the Americal Chemical Society $130(2008)$ 8690-8695.

[19] K. M. Eid, H. Y. Ammar, Adsorption of $\mathrm{SO}_{2}$ on Li Atoms Deposited on $\mathrm{MgO}(100)$ Surface: DFT Calculations, Applied Surface Science 257 (2011) 6049-6058.

[20] K. M. Eid, H. Y. Ammar, A Density Functional Study of $\mathrm{NO}_{2}$ Adsorption on Perfect and Defective $\mathrm{MgO}(100)$ and $\mathrm{Li} / \mathrm{MgO}(100)$ Surfaces, Applied Surface Science 258 (2012) 7689-7698.

[21] H. Y. Ammar, K. M. Eid, $\mathrm{NO}_{2}$ Interaction with Au Atom Adsorbed on Perfect and Defective $\mathrm{MgO}(100)$ Surfaces: Density Functional Theory Calculations, J. Nanosci. Nanotechnol. 13 (2013) 6660-6671.

[22] M. B. Jensen, L. G. M. Pettersson, O. Swang, U. Olsbye, $\mathrm{CO}_{2}$ Sorption on $\mathrm{MgO}$ and $\mathrm{CaO}$ Surfaces: A Comparative Quantum Chemical Cluster Study, J. Phys. Chem. B 109 (2005) 16774-16781.

[23] P. Rinke, A. Schleife, E. Kioupakis, A. Janotti, C. Roedl, F. Bechstedt, M. Scheffler, C. G. Van de Walle, First-Principles Optical Spectra for Centers in MgO, Physical Review Letters 108.

[24] R. R. Sharma, A. M. Stoneham, Theory of the $f_{s}^{+}$surface centre in mgo, J. Chem. Soc., Faraday Trans. 2: Mol. Chem. Phys. (1976) 913.

[25] A. M. Ferrari, G. Pacchioni, Electronic Structure of F and V Centers on the MgO Surface, Journal of Physical Chemistry 99 (1995) 17010-17018.

[26] J. H. Schulman, W. D. Compton, Color Centers in Solds, Macmillian, New York, 1962.

[27] R. I. Eglitis, $\mathrm{Ab}$ initio calculations of $\mathrm{SrTiO}_{3}, \mathrm{BaTiO}_{3}, \mathrm{PbTiO}_{3}, \mathrm{CaTiO}_{3}, \mathrm{SrZrO}_{3}, \mathrm{PbZrO}_{3}$ and $\mathrm{BaZrO}_{3}$ (001), (011) and (111) surfaces as well as F centers, polarons, KTN solid solutions and Nb impurities therein, Int. J. Mod. Phys. B 28 (2014) 1430009.

[28] D. Ricci, C. D. Valentin, G. Pacchioni, P. V. Sushko, A. L. Shluger, E. Giamello, Paramagnetic Defect Centers at the MgO Surface. An Alternative Model to Oxygen Vacancies, Journal of the Americal Chemical Society 125 (2003) 738-747.

[29] G. Mallia, R. Orlando, C. Roetti, P. Ugliengo, R. Dovesi, $F$ center in LiF: quantum mechanical $a b$ initio investigation of the hyperfine interaction between the unpaired electron at the vacancy and its first seven neighbors, Physical Review B 63 (2001) 235102.

[30] C. S. Ewig, J. Tellinghuisen, M. H. Mendenhall, Ab Initio F-center Electron Topology in LiF, Chemical Physics Letters 188 (1992) 501-509.

[31] R. F. W. Bader, J. A. Platts, Characterization of an F-center in an Alkali Halide Cluster, Journal of Chemical Physics 107 (1997) 8545-8553.

[32] J. Carrasco, N. Lopez, F. Illas, On the convergence of isolated neutral oxygen vacancy and divacancy properties in metal oxides using supercell models, Journal of Chemical Physics 122 (2005) 224705.

[33] F. Karsai, P. Tiwald, R. Laskowski, F. Tran, D. Koller, S. Grfe, J. Burgdrfer, L. Wirtz, P. Blaha, F center in lithium fluoride revisited: Comparison of solid-state physics and quantum-chemistry approaches, Physical Review B 89 (2014) 125429.

[34] G. Chen, Z. F. Liu, X. G. Gong, Ab initio study on structural and electronic properties of $\mathrm{ba}_{n} \mathrm{O}_{m}$ clusters, Journal of Chemical Physics (2004) 8020. 
[35] C. F. Williams, J. M. Herbert, Influence of structure on electron correlation effects and electron-water dispersion interactions in anionic water clusters, Journal of Physical Chemistry A 112 (2008) 6171-6178.

[36] E. Zurek, P. P. Edwards, R. Hoffmann, A molecular perspective on lithium-ammonia solutions, Angewandte Chemie, International Edition 48 (2009) 8191-8232.

[37] A. E. Reed, R. B. Weinstock, F. Weinhold, Natural population analysis, Journal of Chemical Physics 83 (1985) 735-746.

[38] J. Gräfenstein, D. Cremer, An efficient algorithm for the density-functional theory treatment of dispersion interactions, Journal of Chemical Physics 130 (2009) 124105.

[39] R. F. W. Bader, Atoms In Molecules A Quantum Theory, Oxford University Press, Oxford, 1990.

[40] G. K. H. Madsen, C. Gatti, B. B. Iversen, L. Damjanovic, G. D. Stucky, V. I. Srdanov, F center in sodium electrosodalite as a physical manifestation of a non-nuclear attractor in the electron density, Physical Review B 59 (1999) 12359.

[41] P. Mori-Sńchez, J. M. Recio, B. Silvi, C. Sousa, A. Martín Pendás, V. L. na, F. Illas, Rigorous characterization of oxygen vacancies in ionic oxides, Physical Review B 66 (2002) 075103.

[42] A. D. Becke, K. E. Edgecombe, A simple measure of electron localization in atomic and molecular systems, Journal of Chemical Physics 92 (9) (1990) 5397-5403.

[43] B. G. Janesko, G. Scalmani, M. J. Frisch, How Far Do Electrons Delocalize?, Journal of Chemical Physics 141 (2014) 144104.

[44] B. G. Janesko, G. E. Scuseria, Local hybrid functionals based on density matrix products, Journal of Chemical Physics 127 (2007) 164117.

[45] A. D. Becke, A real-space model of nondynamical correlation, Journal of Chemical Physics 119 (2003) 2972-2977.

[46] A. D. Becke, Real-space post-Hartree-Fock correlation models, Journal of Chemical Physics 122 (2005) 064101.

[47] E. Proynov, F. Liu, J. Kong, Analyzing effects of strong correlation within Kohn-Sham density functional theory, Physical Review A 88 (2013) 032510.

[48] B. G. Janesko, K. R. Wiberg, G. Scalmani, M. J. Frisch, Electron delocalization range in atoms and on molecular surfaces, Journal of Chemical Theory and Computation 12 (2016) 3185-3194.

[49] B. G. Janesko, G. Scalmani, M. J. Frisch, Quantifying Solvated Electrons' Delocalization, Physical Chemistry Chemcial Physics 17 (2015) 18305-18317.

[50] B. G. Janesko, G. Scalmani, M. J. Frisch, Quantifying electron delocalization in electrides, Journal of Chemical Theory and Computation 12 (2016) 79-91.

[51] B. G. Janesko, Left-right correlation in coupled F-center defects, Journal of Chemical Physics 145 (2016) 054703.

[52] Gaussian Development Version, Revision H.35, M. J. Frisch, G. W. Trucks, H. B. Schlegel, G. E. Scuseria, M. A. Robb, J. R. Cheeseman, G. Scalmani, V. Barone, B. Mennucci, G. A. Petersson, H. Nakatsuji, et. al., Gaussian, Inc., Wallingford CT, 2010.

[53] P. Hohenberg, W. Kohn, Inhomogeneous electron gas, Physical Review 136 (1964) B864-B871.

[54] W. Kohn, L. Sham, Self-consistent equations including exchange and correlation effects, Physical Review 140 (1965) A1133-A1138.

[55] S. H. Vosko, L. Wilk, M. Nusair, Accurate spin-dependent electron liquid correlation energies for local spin density calculations: Critical analysis, Can. J. Phys. 58 (8) (1980) 1200-1211.

[56] J. P. Perdew, K. Burke, M. Ernzerhof, Generalized Gradient Approximation Made Simple, Physical Review Letters 77 (1996) 3865-3868.

[57] A. D. Becke, Density-functional exchange-energy approximation with correct asymptotic behavior, Physical Review A 38 (1988) 3098-3100.

[58] C. Lee, W. Yang, R. G. Parr, Development of the Colle-Salvetti Correlation-Energy Formula Into Functional of the Electron Density, Physical Review B 37 (1988) 785-789.

[59] A. D. Becke, Density-Functional Thermochemistry. III. The Role of Exact Exchange, Journal of Chemical Physics 98 (7) (1993) 5648-5652.

[60] W. J. Stevens, H. Basch, M. Krauss, Journal of Chemical Physics 81 (1984) 6026. 
[61] A. J. Cohen, R. G. Gordon, Theory of the lattice energy, equilibrium structure, elastic constants, and pressure-induced phase transitions in alkali-halide crystals, Physical Review B 12 (1975) 3228.

[62] R. B. Gordon, Color Centers in Crystals, American Scientist 47 (1959) 361-375.

[63] P. Tiwald, F. Karsai, R. Laskowski, S. Grfe, P. Blaha, J. Burgdrfer, L. Wirtz, Ab initio perspective on the Mollwo-Ivey relation for centers in alkali halides, Physical Review B 92 (2015) 144107.

[64] M. Hartmann, J. Pittner, V. Bonačić-Koutecký, Ab initio adiabatic dynamics involving excited states combined with wigner distribution approach to ultrafast spectroscopy illustrated on alkali halide clusters, Journal of Chemical Physics 114 (2001) 2106.

[65] R. S. C. Smart, P. J. Jennings, Particle Size Effects on the Energies of Bulk and Surface F-centers, Trans. Faraday Soc. 67 (1971) 1193.

[66] A. Ruzsinszky, J. P. Perdew, G. I. Csonka, O. A. Vydrov, G. E. Scuseria, Spurious fractional charge on dissociated atoms: Pervasive and resilient self-interaction error of common density functionals, Journal of Chemical Physics 125 (2006) 194112.

[67] E. R. Johnson, A. Otero-de-la Roza, S. G. Dale, Extreme density-driven delocalization error for model solvated-electron system, Journal of Chemical Physics 139 (2013) 184116.

[68] A. J. Cox, J. G. Louderback, S. E. Apsel, L. A. Bloomfield, Magnetism in 4d-transition metal clusters, Physical Review B 49 (1994) 12295.

[69] G. Pacchioni, A. M. Ferrari, E. Giamello, Cluster models of $\mathrm{O}_{2}^{-}$adsorption on regular and defect sites and $\mathrm{F}_{s}$ centers of the $\mathrm{MgO}(100)$ surface, Chemical Physics Letters 255 (1996) 58-64.

[70] E. Giamello*, M. C. Paganini, D. M. Murphy, A. M. Ferrari, G. Pacchioni, A Combined EPR and Quantum Chemical Approach to the Structure of Surface $\mathrm{F}_{s}^{+}(\mathrm{H})$ Centers on MgO, Journal of Physical Chemistry B 101 (1997) 971-982.

[71] M. Chiesa, E. Giamello, D. M. M. andGianfranco Pacchioni, M. C. Paganini, R. Soave, Z. Sojka, Reductive Activation of the Nitrogen Molecule at the Surface of 'Electron-Rich' $\mathrm{MgO}$ and $\mathrm{CaO}$. The $\mathrm{N}_{2}^{-}$Surface Adsorbed Radical Ion, Journal of Physical Chemistry B 105 (2001) 497-505.

[72] D. Ricci, G. Pacchioni, P. V. Sushko, A. L. Shluger, Reactivity of $\left(\mathrm{H}^{+}\right)\left(\mathrm{e}^{-}\right)$Color Centers at the $\mathrm{MgO}$ surface: Formation of $\mathrm{O}_{2}^{-}$and $N_{2}^{-}$Radical Anions, Surface Science 542 (2003) 293-306. 